\section{Fettsäuren-Supplement bessert atopisches Ekzem}

Der Einfluss von Omega-3-Fettsäuren auf das atopische Ekzem wird kontrovers diskutiert. Eine aktuelle kleine Studie attestiert Docosahexaensäure jetzt günstige immunmodulatorische Eigenschaften.

D ocosahexaensäure (DHA) ist eine langkettige, mehrfach ungesättigte Fettsäure des Omega-3-Typs. In einer randomisierten doppelblinden kontrollierten Untersuchung wurde überprüft, ob die orale Gabe von DHA die Immunreaktion von Patienten mit atopischem Ekzem beeinflussen und den Hautzustand verbessern kann. Dazu erhielten 21 Patienten acht Wochen lang DHA-Kapseln, die Kontrollgruppe mit 23 Patienten hingegen eine isokalorische Menge an mittelkettigen, gesättigten Fettsäuren. Vor, während, unmittelbar nach sowie 20 Wochen nach der Supplementierung wurden der klinische Hautbefund sowie immunologische Parameter erhoben.

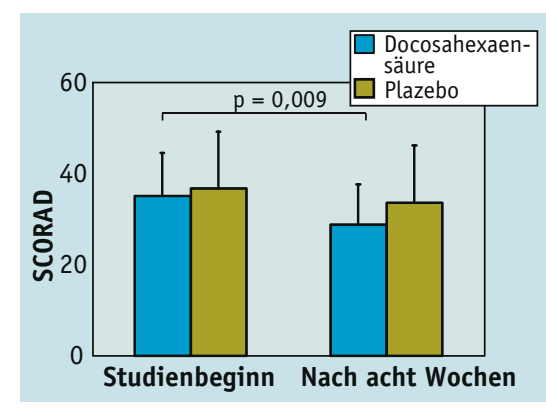

\section{Veränderung des SCORAD}

Die Ergebnisse belegen günstige Eigenschaften der DHA, berichtete Sabine Dölle, Berlin: Im Verlauf der Intervention verbesserte sich in der DHA-Gruppe der SCORAD signifikant (Abb.).
Unter DHA verringerten sich außerdem die IgE-Produktion stimulierter Zellen sowie die Expression verschiedener Zellaktivierungsmarker auf Zellen des peripheren Blutes signifikant. Die Synthese antiinflammatorisch wirksamer Zytokine wurde unter DHA-Supplementierung gesteigert. Auch der Gesamt-Omega-3Fettsäurenspiegel erhöhte sich signifikant. Das Verhältnis von Omega-6- zu Omega-3-Fettsäuren reduzierte sich unter DHA von 2,4 auf 0,9, in der Kontrollgruppe von 2,2 auf 1,6.

Offenbar wirkte sich die Aufklärung der Teilnehmer über die mögliche Bedeutung der Omega-3-Fettsäuren zu Beginn der Studie auch auf die alltägliche Ernährung aus. Dies könnte zumindest gewisse Veränderungen erklären, die auch in der Kontrollgruppe beobachtet wurden, spekulierte Dölle abschließend. abd

Dölle S, Koch C, Rasche C, Jungclas H, Renz H, Worm M: Einfluss von Fettsäurensupplementierungen auf die allergische Immunantwort. 18. Mainzer AllergieWorkshop, Mainz, 10.-11. März 2006 